Technical Communication

\title{
Wetting Properties of Aluminium Surface Structures Fabricated Using Direct Laser Interference Patterning with Picosecond and Femtosecond Pulses
}

\author{
Stephan Milles*1, Johannes Dahms ${ }^{1}$, Bogdan Voisiat ${ }^{1,2}$, Simonas Indrišiūnas², Gediminas Račiukaitis ${ }^{2}$ and Andrés Fabián \\ Lasagni $^{1,3}$ \\ ${ }^{1}$ Institute for Manufacturing Technology, Technische Universität Dresden, George-Baehr-Str. 3c, \\ 01069 Dresden, Germany \\ ${ }^{2}$ Center for Physical Sciences and Technology, Savanoriu Ave. 231, 02300 Vilnius, Lithuania \\ ${ }^{3}$ Fraunhofer-Institut für Werkstoff- und Strahltechnik IWS, Winterbergstr. 28, 01277 Dresden, Ger- \\ many \\ ${ }^{*}$ Corresponding author's e-mail: Stephan.milles@tu-dresden.de
}

\begin{abstract}
In this work, we report about the fabrication of textured aluminium surfaces using Direct Laser Interference Patterning with picosecond (70 ps) and femtosecond (400 fs) laser pulses as well as their wetting properties. The structuring process was performed by varying the pulse numbers from 25 to 250 , resulting in various depths ranging from $0.9 \mu \mathrm{m}$ to $6.8 \mu \mathrm{m}$. The wetting analysis shows that the ps-patterned surfaces exhibit long-term superhydrophobic characteristics (at $21^{\circ} \mathrm{C}$ and $16 \%$ air humidity). Differently, for the fs-processed substrates, a hydrophobic character was firstly observed, which later (after 16 days) dropped to contact angles similar as the untreated material.
\end{abstract}

DOI: 10.2961/jlmn.2021.01.3001

Keywords: direct laser interference patterning, picosecond, femtosecond, aluminium 2024, wettability, superhydrophobicity

\section{Introduction}

Superhydrophobic surfaces are economically highly interesting since they are related to additional functionalities like corrosion-resistance, anti-icing, or the ability of selfcleaning [1]. There are a wide variety of chemical and physical technologies existing to fabricate these water repellent surfaces. They are ranging from CVD and PVD treatments, over chemical etching to expensive lithographical or wearintensive micro-milling processes [2-5].

Alternative approaches to the disadvantageous existing manufacturing processes are laser texturing methods, as Direct Laser Interference Patterning (DLIP). By using this onestep fabrication method, a single laser beam is divided into at least two coherent sub-beams, which are then superimposed and focused on the surface, resulting in a periodic laser light intensity distribution [6-10].

DLIP has been proven as a suitable tool to fabricate microstructures on aluminium surfaces providing superhydrophobic, ice-repellent, and self-cleaning functionalities [1115]. The wetting characteristic of a surface besides the chemistry is mainly controlled by the topographical condition. Typically, the surface topography is defined by roughness parameters, such the root mean square height $\left(\mathrm{S}_{\mathrm{q}}\right)$ or the maximum height $\left(S_{z}\right)$ [16]. It has been known for some time that an increase of the roughness of hydrophilic surfaces (contact angle $<90^{\circ}$ ) results in hydrophilic and hydrophobic surfaces becoming more hydrophobic (contact angle > $\left.90^{\circ}\right)[17,18]$. The surface roughness strongly depends on the laser processing parameters, among them is the pulse duration. In the past, picosecond (ps) pulsed laser sources were preferred for microstructuring since they offer a suitable compromise between sufficiently high ablation rate and simultaneously reduced melting effects compared to nanosecond pulsed ablation processes, where melting is the dominant ablation phenomenon. Significantly more precise structures can be fabricated using femtosecond (fs) pulsed laser sources due to the reduction of thermal losses on the material surface [19].

On the other hand, this "cold ablation" also leads to reduced oxide formation on the surface and thus to fewer attachment points for organic components. These are adsorbed onto the surface by the surrounding atmosphere after ps irradiation, for example and are responsible for a superhydrophobic characteristic [20]. This functionality is also very interesting as it can prevent biofouling, contribute to drag reduction, or be used in the field of oil and water separation [21]. However, it is still not clear, which pulse duration (ps or fs) provides better structuring results regarding a functional superhydrophobic surface when DLIP technology is applied. The dominant ablation phenomena can be described as ultra-fine ablation for both cases [22]. However, especially on materials with a very low melting temperature like aluminium $\left(660^{\circ} \mathrm{C}\right)$, the differences between picosecond and femtosecond ablation are tremendous.

In this publication, the results of multi-pulse ultrashortpulse laser ablation on aluminium 2024 are discussed. A four-beam DLIP technology, in combination with 70 ps and $400 \mathrm{fs}$ laser, has been applied to fabricate the microstructures with a period of $3.8 \mu \mathrm{m}$. The number of pulses was varied between 25 to 250, resulting in the fabricated structure depths ranging from $0.9 \mu \mathrm{m}$ to $6.8 \mu \mathrm{m}$. The produced patterns were investigated regarding their surface topography 
and their wetting characteristic for a period of time of 35 days after processing.

\section{Materials and methods \\ 2.1 Material}

Plates of Aluminium clad (Al 2024) with lateral dimensions of $100 \mathrm{~mm} \times 100 \mathrm{~mm}$ and $1 \mathrm{~mm}$ thickness were used in this study. The samples were electrolytically polished resulting in an initial surface roughness of $\left(\mathrm{S}_{q}\right)$ of $41 \mathrm{~nm} \pm 10$ $\mathrm{nm}$. Before laser treatment the samples were cleaned from contamination with isopropanol in an ultrasonic bath for 10 $\min$ at $22^{\circ} \mathrm{C}$. Aluminum is naturally covered with an aluminium oxide layer which is usually $20-30 \mathrm{~nm}$ thick $[23,24]$.

\subsection{Picosecond DLIP}

Aluminium 2024 sheets were structured using a DLIP$\mu$ Fab workstation, developed by the TU Dresden and Fraunhofer IWS. It utilizes a solid-state 70 ps laser (neoLASE $\mathrm{GmbH}$, Hannover, Germany) emitting a $532 \mathrm{~nm}$ wavelength with a nominal output power of $2.7 \mathrm{~W}$ at a repetition rate of $10 \mathrm{kHz}$ resulting in laser fluence of $1.4 \mathrm{~J} / \mathrm{cm}^{2}$. The beam quality of the applied ps laser source regarding the $\mathrm{M}^{2}$ was $<1.3$ for a Gaussian beam profile and the laser radiation was linearly polarized with a polarization ratio $>100: 1$. For the fabrication of dot-like patterns, a four-beam DLIP was applied with a spatial period of $3.8 \mu \mathrm{m}$. The height of the interference volume in which a periodic energy distribution is present was about $40 \mu \mathrm{m}$. A detailed description of the used setup was already published elsewhere [25].

\subsection{Femtosecond DLIP}

Femtosecond DLIP processing was performed using a workstation, developed by the FTMC, ELAS Ltd. and Amplight KG in which high pulse energy femtosecond laser (200 fs - 10 ps, $1 \mathrm{kHz}, 1030 \mathrm{~nm}, 14 \mathrm{~mJ}$ ), provided by Amplight KG, can be managed. The optical setup consisted of a diffractive optical element for beam splitting and a $4 \mathrm{f}$ lens setup for the collection of separate beams. This setup provides a simple way to overlap ultrashort laser pulses when using the interference technique accurately and contains no dispersive elements, that can distort temporal (and spatial) pulse characteristics [26]. Using the diffractive optical element from Holo/Or with a separation angle of $7.71^{\circ}$, the laser beam was split into four beams (plus zero-order beam which was not used). This is the angle at which the beams are separated in after passing the DOE. By transmitting these beams through the $\mathrm{x} 2$ magnification $4 \mathrm{f}$ lens setup, they are overlapped on the samples surface, that interfere at the angle of $15.6^{\circ}$, forming the interference period of $3.8 \mu \mathrm{m}$ with the $1030 \mathrm{~nm}$ wavelength. The pulse was stretched after passing all optical elements resulting in in a constant pulse duration of $400 \mathrm{fs}$ at the sample surface. It was measured using Frequency-Resolved Optical Gating Pulse Analyzer (FROG).

The diameter of the interference spot, measured using a beam profiler camera at $1 / \mathrm{e}^{2}$ level, was $2.3 \mathrm{~mm}$. However, the ablation threshold was exceeded in slightly smaller areas with a diameter of approximately $2 \mathrm{~mm}$. Considering the interference angle $\left(15.6^{\circ}\right)$ and the diameter of the laser beams $(2.3 \mathrm{~mm})$ at the material' surface, it is possible to calculate the height of the interference volume, corresponding to $16.8 \mathrm{~mm}$. On the sample surface, $1.3 \mathrm{~J} / \mathrm{cm}^{2}$ fluence was achieved using the maximal available pulse energy. The beam quality of the applied fs laser source regarding the $\mathrm{M}^{2}$ was $<1.3$ for a Gaussian beam profile and the laser radiation is linearly polarized with a polarization ratio $>100: 1$. The polarization direction at the optical systems' input can be random because it does not influence the interference intensity profile due to a relatively small interference angle of $15.6^{\circ}[27]$.

\subsection{Surface characterization}

For the analysis of the surface topography and structure depth, a confocal microscope (Sensofar S Neox, Terrassa, Spain) with a 150x objective was used, providing vertical and lateral resolution of $2 \mathrm{~nm}$ and $140 \mathrm{~nm}$, respectively. The laser-treated substrates were also evaluated using a scanning electron microscope (ZEISS Supra 40 VP, Carl Zeiss Microscopy GmbH, Jena, Germany) at the operating voltage of $5.0 \mathrm{kV}$

\subsection{Wettability characterization}

The wettability of the patterned surface was analyzed performing static water contact angle measurements over 35 days period with the drop shape analyzer (DSA 100 S, Krüss GmbH, Hamburg, Germany), frequently measuring every three days. The tests were performed using $4 \mu \mathrm{l}$ deionized water droplets at ambient conditions of $21^{\circ} \mathrm{C}$ and $16 \%$ air humidity. For the water contact angle measurement, the tangent droplet fitting method was applied. Each measurement was repeated at least five times for statistical purposes.

\section{Results and discussion \\ 3.1 Laser material processing}

The aluminium samples were structured using the fourbeam interference configuration with the fs and ps laser sources. In the case of the fs laser patterning, the interference spot diameter formed by an optical imaging system was relatively large $(2300 \mu \mathrm{m})$. Therefore, the maximum laser power was utilized to have the laser fluence $\left(1.3 \mathrm{~J} / \mathrm{cm}^{2}\right)$ high enough to ablate the aluminium surface. Thereby, experiments performed with the ps laser source were conducted using the same laser fluence but with a smaller interference spot (45 $\mu \mathrm{m}$ in diameter). In addition, the number of laser pulses per spot was varied between 25 and 250. The spots were positioned in such a way that the patterned areas overlap only by $10 \%$. The spatial period of the hole-like structures, which is described as the distance between the centers of two holes, was for both applied methods, ps and fs processing, constant $3.8 \mu \mathrm{m}$.

Because of the small overlap used (10\%), there is no need to carefully align the spots so that the ablated craters of the overlapping areas are precisely superimposed with each other. The SEM micrograph of the structures fabricated with a low (50) and high (250) number of fs and ps pulses are shown in Fig. 1. The structures fabricated by four-beam DLIP consists of periodically distributed craters, ablated at the interference intensity peaks. It has to be noted that the interference spot exhibits a Gaussian intensity envelope, which results in dissimilar structure heights, following the intensity distribution. For allowing a comparison between the experiments, the micrographs were taken at the center of laser spots, where the peak intensity is the highest. 
A first pronounced difference between the ps- and fs-patterned structures is seen in Fig. 1a, and Fig. 1c, where the structures fabricated at a low number of pulses are shown. In the case of the fs patterning (Fig. 1c), the craters are almost perfectly round, having no rims around the edges. However, in the case of ps pulses (Fig. 1a), not all craters maintain their shapes, because of the irregular formation of the rims, which surround them. Formation of the rims confirms the appearance of the melted material inside the ablation zones (craters), which is redeposited around the craters through Marangoni convection and recast pressure [28]. Because the molten material removed from the crater's center is brought on the surface of the untreated area between the craters, it contributes highly to the final depth of the structure. For the picosecond pulsed structures, the morphological appearance between 50 and 250 pulses (Fig.1a, b) is not significantly different. In contrast, distinctly larger craters are observed when the number of femtosecond pulses is increased from 50 to 250 (Fig. 1d).

Also, the formation of the laser-induced periodic surface structures (LIPSS) with a period of $~ 600 \mathrm{~nm}$ was observed in the areas between the ablated craters. Since these structures are significantly smaller than the laser wavelength used, they can be assigned to the classification of high spatial frequency LIPSS [29]. In the case of ps laser-ablated structures, the LIPSS features were not observed, possible due to the big amount of recast material redeposited on the surface between the craters. Compared to previous studies where LIPSS have been observed in aluminum using DLIP [11] or direct laser writing with multi-pulse texturing [30,31], the LIPSS features were observed for fluences between $0.10 \mathrm{~J} / \mathrm{cm}^{2}$ and $0.17 \mathrm{~J} / \mathrm{cm}^{2}$, which is significantly much lower than the used fluence of $1.4 \mathrm{~J} / \mathrm{cm}^{2}$ in our experiments [32].

In addition to the surface morphology, Fig. 2 shows the structure depth evolution as a function of the number of laser pulses. The structure depth was measured using confocal microscopy, as described in the experimental section. It can be seen that the structures fabricated with ps pulses are up to 2.2 times deeper than the fs-fabricated structures for 250 pulses $(6.8 \mu \mathrm{m}$ vs. $3.1 \mu \mathrm{m})$. This result can be explained by the stronger melt ejection from the craters, which are ablated using ps pulses, resulting in ridge formation [33]. Remarkably, structure depth of $4.8 \mu \mathrm{m}$ can be achieved using only 25 pulses in case of the ps laser source, whereas the topography of the patterns created with the fs laser exhibit only $0.8 \mu \mathrm{m}$ in depth when using the same number of pulses.

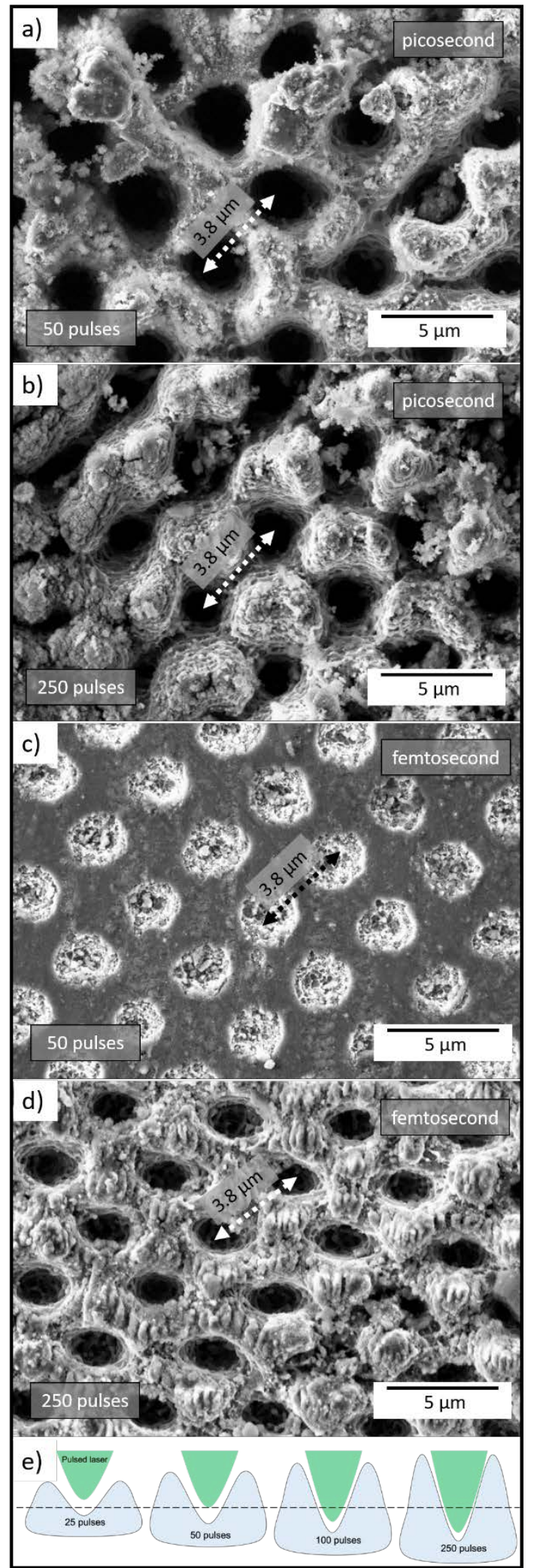

Fig. 1 SEM images of hole-like microstructures with a spatial period of $3.8 \mu \mathrm{m}$ on aluminium 2024 irradiated with $532 \mathrm{~nm}$ wavelength, a pulse duration of $70 \mathrm{ps}, 10 \mathrm{kHz}$ repetition rate and laser fluence of $1.4 \mathrm{~J} / \mathrm{cm}^{2}(\mathrm{a}, \mathrm{b})$ and with $1030 \mathrm{~nm}$ wavelength, a pulse duration of $400 \mathrm{fs}$ (c, d) $1 \mathrm{kHz}$ repetition rate and laser fluence of $1.3 \mathrm{~J} / \mathrm{cm}^{2}$ applying 50 pulses (a, c) and 250 pulses (b, d). The scheme in (e) illustrates the pile-ups of the material with an increasing number of pulses relative to the origin surface (dashed line). 


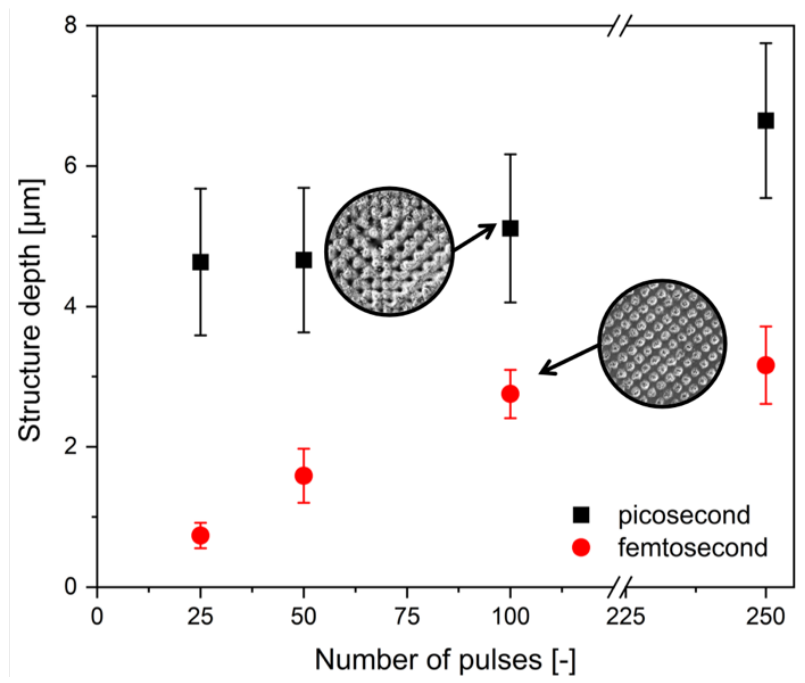

Fig. 2 Structure depth as a function of the number of used pulses for 70 ps (black) and 400 fs (red) irradiation. The circular insets depict the exemplarily micro-structured surface patterned with 100 pulses.

\subsection{Long-term wetting studies}

After laser processing, the wetting characteristic of the fabricated microstructures was analyzed by means of static water contact angle (WCA) measurements over a period of 35 days. The resulting evolution is presented in Fig 3.

The WCA of the untreated surface reference stays at a level between $88^{\circ}$ and $104^{\circ}$ (grey bar). Immediately after the laser processes (day 1-3), the structures showed a hydrophilic condition, expressed by a WCA under $90^{\circ}$, independently of the applied pulse duration or number of pulses. Mainly responsible for this behavior are unsaturated aluminum and oxygen atoms leading to a hydrophilic condition, which has already been reported in other works [34,35].

In the following, the WCA for all picosecond-treated substrates was constantly rising with time up to superhydrophobic (WCA $>150^{\circ}$ ) condition after 16 days, where it remained constant until day 32. Furthermore, no significant differences were observed regarding the final static contact angles among the surfaces fabricated using different numbers of ps pulses (Fig. 3a). In addition, the microstructures fabricated with 250 pulses, featured with the largest structure depth of $6.8 \mu \mathrm{m}$, presented in all measurements of the last 16 days the highest WCA. A surface texture with a minimum depth of $4.7 \mu \mathrm{m}$ is primarily responsible for the superhydrophobic characteristic since the minimum roughness for an increasing wetting state is existing. Moreover, due to the symmetrical patterning, hence the systematic formation of laser-induced melt residues, the fabricated texture provides a controllable roughness, which specifically determines the wetting behavior $[11,36]$. The required minimum roughness was fabricated only by using picosecond pulsed irradiation. DLIP structures on a surface allow a homogeneous and uniform modification of a topography, which allows a constant functionality concerning the structure. When wetting surfaces, the aspect ratio of the textures has an indirect influence. In order to achieve a superhydrophobic condition, a Cassie-Baxter wetting is aspired, where a single droplet is exclusively in contact with the roughness peaks of a topography $[17,37]$.
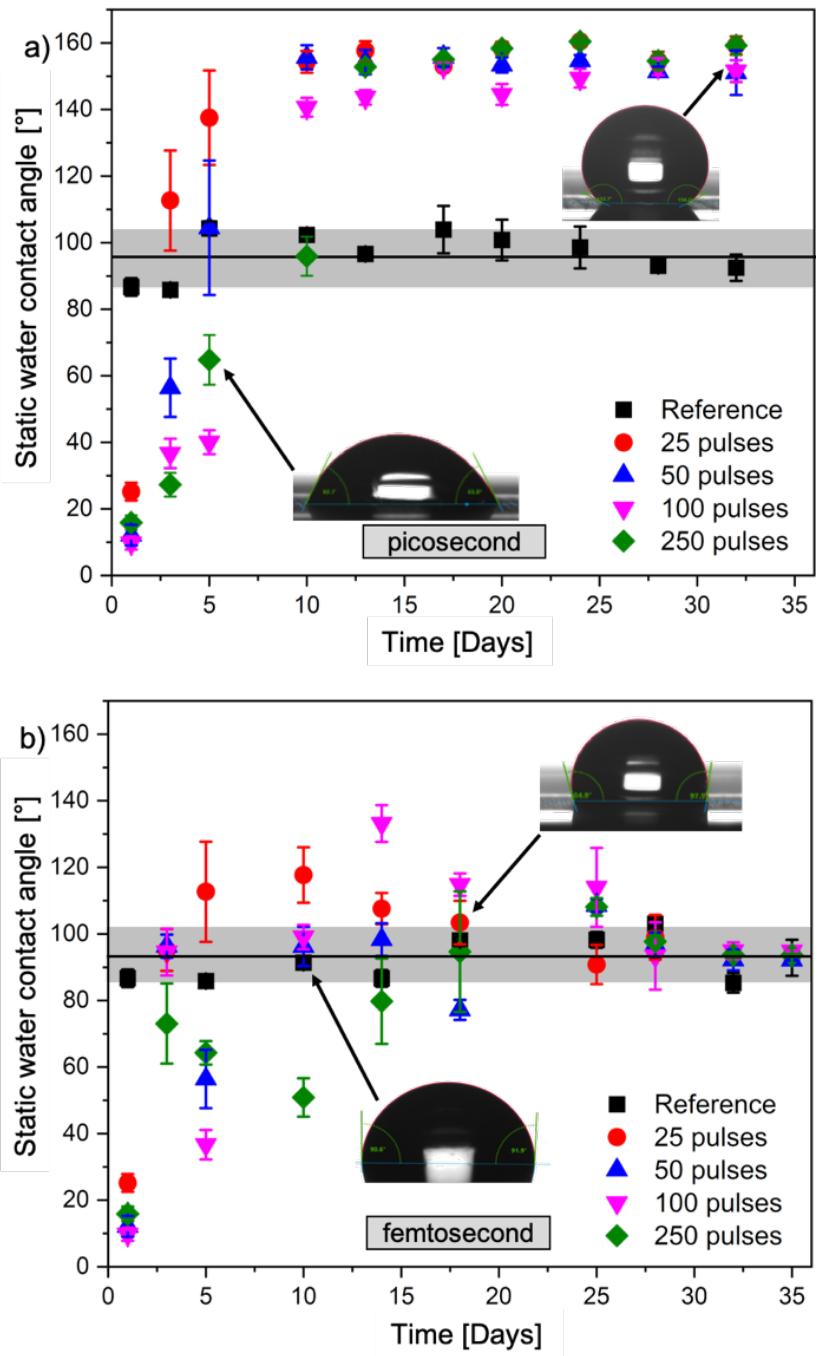

Fig. 3 Static water contact angle (WCA) measurements as a function of time after laser processing, measured with deionized water droplets of $4 \mu \mathrm{l}$ volume for the structures fabricated with 25, 50, 100, 250 pulses, for picosecond (a) and femtosecond (b) pulse durations. The photographic insets represent typical appearances of the droplet shape.

This state is most likely to be achieved when the structure has a high aspect ratio. Furthermore, the temperature of the environment, the surface tension of the liquid used for the droplets and the surface chemistry influence the wetting state $[38,39]$. It was also shown that a texturing with a pattern in the hundred $\mu \mathrm{m}$ range produces a more hydrophobic surface despite micrometer deep structures [31]. However, a utterly superhydrophobic property has so far only been achieved with significantly smaller structures or by additional chemical treatment [40].

In contrast, the femtosecond-processed samples showed a similar rising trend but reaching a maximal WCA of $131^{\circ}$ after 14 days. However, this trend did not stay constant after 25 days. The WCA of all structures dropped down to $92^{\circ}$, which is very similar to the wetting state of the untreated reference. No clear trend could be seen, independently of the applied number of pulses (Fig. 3b).

It is known that the wetting characteristic of a surface is defined by two influencing factors: The surface chemistry and the roughness, which is expressed in this study by the structure depth. The chemistry is not considered in this case since 
it is well known that carbon from the ambient air is accumulated subsequently on aluminium that has been irradiated with the laser. This carbon content then increases the number of non-polar sites on the surface, which leads to an increase of the static water contact angle. In combination with a surface texture, it results in a constant superhydrophobic characteristic $[12,41]$. This effect is dominant in ns and ps laser treatment $[20,42,43]$. On the other hand, it is well known that an increase of aluminium oxide, formed due to laser processing, is responsible for a large number of polar-sites on the surface. These unsaturated aluminium and oxygen atoms can be responsible for the decrease of the maximum WCA of $131^{\circ}$ after 14 days, leading to a hydrophilic condition [35,44,45] (Fig. 3b).

In the fs section of this study, the maximum structure depths of $3.1 \mu \mathrm{m}$ and the slightly more dominant aluminium oxide deposition on the surface lead to an approximation to the reference wetting state with a WCA of $93^{\circ}$ after 35 days (Fig. 3b). It was also reported, that fs processed aluminium substrates reached a constant superhydrophobic condition, if their topography fulfilled a certain minimum roughness [4649]. Furthermore, it is the state of the art that a nano roughness alone can make a surface more hydrophobic, but not superhydrophobic [50]. This is achieved either by an additional micro-roughness or by applying further silane-based solution. Such coating reduces the surface free energy and thus the wetting of water, and therefore it increases the water contact angle [51].

The chemical phenomena on a laser-treated Aluminium surface have already been investigated in detail in the past and are summarized briefly below, both for ps and fs treatment. For example, a micro-nanostructure with a roughness of up to $5.2 \mu \mathrm{m}$ was generated on pure aluminium (99.9\% purity) by means of $10 \mathrm{ps}$ irradiation. XPS was used to detect an increased occurrence of non-polar groups, which were responsible for a final water contact angle of up to $153^{\circ}$ [20]. Similar results were reported for line-like structures on the alloy Al 5052, which were produced with 500 ps radiation. The up to $66 \mu \mathrm{m}$ deep structures contained $\mathrm{C}$ on the surface, which indicates carboxylic acids as demonstrated by EDX and XPS analysis [52]. Liu et al. also showed that fs irradiation on Aluminium after 60 days leads to an increase in the $\mathrm{C} / \mathrm{Al}$ atomic ratio at the surface and thus to a superhydrophobic characteristic. However, this is only the case if the corresponding topography exceeds a minimum roughness of several $\mu \mathrm{m}$ [49]. Different wetting states exhibiting in the first 17 days on fs processed samples, could be a result of short-term change in surface chemistry. However, for a longterm transition into the superhydrophobic regime, a topography with a significantly increased roughness is required, as in the case of ps fabricated structures.

\section{Conclusion}

In the present study, the influence of pico- and femtosecond irradiation on the DLIP process of aluminium 2024 was studied. The resulting microstructures were fabricated employing four-beam DLIP setup and investigated regarding their surface topography and their wetting properties. It was shown that the ultra-fine ablation is dominant for both pulse duration regimes. Femtosecond irradiation is suitable for precise structures with a maximum depth of $3.1 \mu \mathrm{m}$, while picosecond irradiation allows fabricating microstructures with a structure depth up to $6.8 \mu \mathrm{m}$, which finally, developed a constant superhydrophobic characteristic with water contact angles above $150^{\circ}$ after 16 days. This is consistent with the current state of the art. According to that, an increased roughness or texture depth contributes to the superhydrophobic Cassie-Baxter condition. This roughness was created by the ps texturing due to the strong redeposition of the recast material. Contrarily, for the fs treated samples, the limited structure depth of $3.1 \mu \mathrm{m}$ and the compensating chemical processes on the surface did not lead to a significant change of the wetting behavior in the long-term observation.

\section{Acknowledgments}

This work was carried out in the framework of the Reinhart Koselleck project (323477257), which has received funding from the German Research Foundation (German: Deutsche Forschungsgemeinschaft DFG). The work of A.L. is also supported by the German Research Foundation (DFG) under the Excellence Initiative program by the German federal and state government to promote top-level research at German universities. The work of B.V. and S.I. was carried out in the framework of SLAM-HELP project, which was supported by EUREKA and the European Commission under the Eurostars program.

\section{References}

[1] M. Okoshi, Y. Awaihara, T. Yamashita, and N. Inoue: Mater. Lett., 139, (2015) 300.

[2] X. Zhang, F. Shi, J. Niu, Y. Jiang, and Z. Wang: J. Mater. Chem., 18, (2008) 621.

[3] S. Li, K. Page, S. Sathasivam, F. Heale, G. He, Y. Lu, Y. Lai, G. Chen, C. J. Carmalt, and I. P. Parkin: J. Mater. Chem., A6, (2018) 17633.

[4] L. Gao, W. Zhou, Y. Wang, S. Wang, C. Bai, S. Li, B. Liu, J. Wang, and Y. L. Li: Optik, 127, (2016) 5211.

[5] E. Williams, E. B. Brousseau, and A. Rees: Int. J. Adv. Manuf. Technol., 74, (2014) 769.

[6] B. Voisiat, M. Gedvilas, S. Indrišiūnas, and G. Račiukaitis: Physcs. Proc., 12, (2011) 116.

[7] S. Alamri, B. Krupop, T. Steege, A. AguilarMorales, V. Lang, S. Storm, F. Schell, C. Zwahr, C. Kracht, M. Bieda, B. Voisiat, U. Klotzbach, A. F. Lasagni, and T. Kunze: Proc. SPIE, Vol. 10906, (2019) 109060S.

[8] S. Indrisiunas, B. Voisiat, A. Žukauskas, and G. Račiukaitis: Proc SPIE, Vol. 9350, (2015) 935003.

[9] D. Sola, C. Lavieja, A. Orera, and M. J. Clemente: Opt. Laser Eng., 106, (2018)139.

[10] A. Rosenkranz, M. Hans, C. Gachot, A. Thome, S. Bonk, and F. Mücklich: Lubricants, 4, (2016) 2.

[11] S. Milles, B. Voisiat, M. Nitschke, and A. F. Lasagni: J. Mater. Process. Tech., 270, (2019) 142.

[12] S. Milles, M. Soldera, B. Voisiat, and A. F. Lasagni: Sci. Rep., 9, (2019) 1.

[13] S. Milles, M. Soldera, T. Kuntze, and A. F. Lasagni: Appl. Surf. Sci., 525, (2020) 146518.

[14] J. T. Cardoso, A. I. Aguilar-Morales, S. Alamri, D. Huerta-Murillo, F. Cordovilla, A. F. Lasagni, and J. L. Ocaña: Opt. Las. Eng., 111, (2018) 193.

[15] D. Huerta-Murillo, A. García-Girón, J. M. Romano, J. T. Cardoso, F. Cordovilla, M. Walker, S. S. 
Dimov, and J. L. Ocaña: Appl. Surf. Sci., 463, (2019) 838.

[16] B. Bhushan: "Surface Roughness Analysis and Measurement Techniques” ed. by CRC Press (Bhushan, Boca Raton, 2000) p.1760.

[17] A. B. D. Cassie and S. Baxter: Trans. Faraday Soc., 40, (1944) 546.

[18] R. N. Wenzel: Ind. Eng. Chem., 28, (1936) 988.

[19] J. Bonse, S. V. Kirner, M. Griepentrog, D. Spaltmann, and J. Krüger: Materials, 11, (2018) 5.

[20] J. Long, M. Zhong, H. Zhang, and P. Fan: J. Colloid Interface Sci., 441, (2015) 1.

[21] A. Samanta, Q. Wang, S. K. Shaw, and H. Ding: Mater. Design, 192, (2020) 108744.

[22] M. Gedvilas, S. Indrišiūnas, B. Voisiat, E. Stankevičius, A. Selskis, and G. Račiukaitis: Phys. Chem. Chem. Phys., 20, (2018) 12166.

[23] I. Pires, L. Quintino, C. M. Rangel, G. E. Thompson, P. Sheldon, and X. Zhou: Int. J. Surf. Sci. Eng., 78, (2000) 179.

[24] J. M. Holt, H. Mindlin, C. Y. Ho: "Structural Alloys Handbook” ed. by J. M. Holt, H. Mindlin, C. Y. Ho, (CINDAS/Purdue University, West Lafayette, 1997) p.1-2500.

[25] S. Eckhardt, M. Siebold, and A. F. Lasagni: Opt. Express, 24, (2016) 553.

[26] A. A. Maznev, T. F. Crimmins, and K. A. Nelson: Opt. Lett., 23, (1998) 1378.

[27] S. Indrisiunas, B. Voisiat, M. Gedvilas, and G. Račiukaitis: Proc. LPM 2017, (2017) 1-5.

[28] A. I. Aguilar-Morales, S. Alamri, T. Kunze, and A. F. Lasagni: Opt. Laser Technol., 107, (2018) 216.

[29] M. Mezera and G. R. B. E. Römer: Opt. Express, 27, (2019) 6012.

[30] J. I. Ahuir-Torres, M. A. Arenas, W. Perrie, and J. de Damborenea: Opt. Las. Eng., 103, (2018) 100.

[31] J. I. Ahuir-Torres, M. A. Arenas, W. Perrie, G. Dearden, and J. de Damborenea: Surf. Coat. Tech., 321, (2017) 279.

[32] P. Hauschwitz, D. Jochcová, R. Jagdheesh, D. Rostohar, J. Brajer, J. Kopeček, M. Cimrman, M. Smrž, T. Mocek, and A. Lucianetti: Opt. Laser Technol., 133, (2021) 106532.

[33] B. Voisiat, C. Zwahr, and A. F. Lasagni: Appl. Surf. Sci., 471, (2019) 1065.

[34] E. Louvis, P. Fox, and C. J. Sutcliffe: J. Mater. Proc. Tech., 211, (2011) 275.

[35] R. Jagdheesh, M. Diaz, and J. L. Ocaña: RSC Adv., 6, (2016) 72933.

[36] R. Baumann, S. Milles, B. Leupolt, S. Kleber, J. Dahms, and A. F. Lasagni: Opt. Las. Eng., 137, (2021) 106364.

[37] A. Giacomello, M. Chinappi, S. Meloni, and C. M. Casciola: Phys. Rev. Lett., 109, (2012) 226102.

[38] B. Bhushan and Y. C. Jung: J. Phys. Condens. Mat. 20, (2008) 225010.

[39] A. Marmur: Ann. Rev. Mater. Res., 39, (2009) 473.

[40] V. Vercillo, J. T. Cardoso, D. Huerta-Murillo, S. Tonnicchia, A. Laroche, J. A. Mayén Guillén, J. L. Ocaña, A. F. Lasagni, and E. Bonaccurso: Mater. Lett., 3, (2019) 100021.
[41] A.-M. Kietzig, S. G. Hatzikiriakos, and P. Englezos: Langmuir, 25, (2009) 4821.

[42] L. R. de Lara, R. Jagdheesh, and J. L. Ocaña: Mater. Lett., 184, (2016) 100.

[43] Z. Lu, P. Wang, and D. Zhang: Corros. Sci., 91, (2015) 287.

[44] G. Azimi, R. Dhiman, H.-M. Kwon, A. T. Paxson, and K. K. Varanasi: Nat. Mater., 12, (2013) 315.

[45] M. M. Gentleman and J. A. Ruud: Langmuir, 26, (2010) 1408.

[46] A.-M. Kietzig, M. N. Mirvakili, S. Kamal, P. Englezos, and S. G. Hatzikiriakos: J. Adhes. Sci. Technol., 25, (2011) 2789.

[47] P. Bizi-bandoki, S. Valette, E. Audouard, and S. Benayoun: Appl. Surf. Sci., 273, (2013) 399.

[48] P. Bizi-Bandoki, S. Benayoun, S. Valette, B. Beaugiraud, and E. Audouard: Appl. Surf. Sci., 257, (2011) 5213.

[49] W. Liu, M. Cai, X. Luo, C. Chen, R. Pan, H. Zhang, and M. Zhong: J. Laser Appl., 31, (2019) 022503.

[50] L. Orazi, I. Gnilitskyi, and A. P. Serro: J. Micro Nano-Manuf., 5, (2017) 021008.

[51] A. I. Gavrilov, D. V. Golovin, A. M. Emelyanenko, D. A. Zayarny, A. A. Ionin, S. I. Kudryashov, S. V. Makarov, P. N. Saltuganov, and L. B. Boinovich: Bull. Russ. Acad. Sci. Phys., 80, (2016) 358.

[52] W. Xing, Z. Li, H. Yang, X. Li, X. Wang, and N. Li: Mater. Design, 183, (2019) 108156.

(Received: May 28, 2020, Accepted: March 14, 2021) 